Paper

\title{
On the embedding constant of the Sobolev type inequality for fractional derivatives
}

\author{
Makoto Mizuguchi ${ }^{1 a)}$, Akitoshi Takayasu ${ }^{2 *}$, Takayuki Kubo ${ }^{3}$, \\ and Shin'ichi Oishi4 \\ ${ }^{1}$ Graduate School of Fundamental Science and Engineering, Waseda University \\ ${ }^{2}$ Research Institute for Science and Engineering, Waseda University \\ ${ }^{3}$ Institute of Mathematics, University of Tsukuba \\ 4 Department of Applied Mathematics, Faculty of Science and Engineering, \\ Waseda University and CREST, JST \\ a) makoto.math@fuji.waseda.jp
}

Received October 5, 2015; Revised March 24, 2016; Published July 1, 2016

Abstract: This paper is concerned with the embedding constant of the Sobolev type inequality for fractional derivatives on $\Omega \subset \mathbb{R}^{N}(N \in \mathbb{N})$. The constant is explicitly described using the analytic semigroup over $L^{2}(\Omega)$ generated by the Laplace operator. Some numerical examples of estimating the embedding constant are also provided.

Key Words: embedding constant, Sobolev inequality, fractional derivative, analytic semigroup

\section{Introduction}

Let $\Omega$ be a domain in $\mathbb{R}^{N}(N \in \mathbb{N})$. For $p \geq 1$, we denote the usual Lebesgue space by

$$
L^{p}(\Omega):=\left\{\begin{array}{l}
\left\{f:\left.\Omega \rightarrow \mathbb{R}\left|\int_{\Omega}\right| f(x)\right|^{p} d x<\infty\right\} \quad(1 \leq p<\infty), \\
\left\{f: \Omega \rightarrow \mathbb{R}\left|\operatorname{ess}_{\sup } \in \Omega\right| f(x) \mid<\infty\right\} \quad(p=\infty)
\end{array}\right.
$$

with the norm

$$
\|f\|_{L^{p}(\Omega)}:= \begin{cases}\left(\int_{\Omega}|f(x)|^{p} d x\right)^{\frac{1}{p}} & (1 \leq p<\infty), \\ {\operatorname{ess} \sup _{x \in \Omega}|f(x)|}(p=\infty),\end{cases}
$$

respectively. Let a function space

$$
H_{0}^{1}(\Omega):=\left\{u \in L^{2}(\Omega) \mid \nabla u \in\left(L^{2}(\Omega)\right)^{2} \text { and } u=0 \text { on } x \in \partial \Omega \text { in the trace sense. }\right\},
$$

where the $L^{2}$ inner product is denoted by $(u, v)_{L^{2}(\Omega)}=\int_{\Omega} u(x) v(x) d x$. Let $A: \mathcal{D}(A) \rightarrow L^{2}(\Omega)$ be an operator defined by

$$
(A u, v)_{L^{2}(\Omega)}=(\nabla u, \nabla v)_{L^{2}(\Omega)}, \forall v \in H_{0}^{1}(\Omega)
$$

*Present affiliation: Faculty of Engineering, Information and Systems, University of Tsukuba 
where $\mathcal{D}(A):=\left\{u \in H_{0}^{1}(\Omega) \mid A u \in L^{2}(\Omega)\right\}$ denotes the domain of $A$. For $i \in \mathbb{N}$, let $\lambda_{i}$ be an eigenvalue $^{1}$ of $A$ satisfying $0<\lambda_{1} \leq \lambda_{2} \leq \cdots$. A function $\psi_{i} \in \mathcal{D}(A)$ denotes an eigenfunction of $A$ corresponding to $\lambda_{i}$ satisfying $\left(\psi_{i}, \psi_{j}\right)_{L^{2}(\Omega)}=\delta_{i, j}$, where $\delta_{i, j}$ is Kronecker's delta ${ }^{2}$. For $u \in L^{2}(\Omega)$, we express $u=\sum_{j=1}^{\infty} c_{j} \psi_{j}$ using the spectral decomposition, where $c_{i}=\left(u, \psi_{i}\right)_{L^{2}(\Omega)}$. Then, since $A: \mathcal{D}(A) \rightarrow L^{2}(\Omega)$ is a positive definite and self-adjoint operator, the fractional power of $A$ is defined by

$$
A^{\alpha} u=\sum_{i=1}^{\infty} \lambda_{i}^{\alpha} c_{i} \psi_{i} \in L^{2}(\Omega)
$$

for $0 \leq \alpha \leq 1$, where $\mathcal{D}\left(A^{\alpha}\right)=\left\{u=\sum_{i=1}^{\infty} c_{i} \psi_{i} \in L^{2}(\Omega) \mid \sum_{i=1}^{\infty} \lambda_{i}^{2 \alpha} c_{i}^{2}<\infty\right\}$ denotes the domain of $A^{\alpha}$. Let us define a function space ${ }^{3} X_{\alpha}$ as $X_{\alpha}=\mathcal{D}\left(A^{\alpha}\right)$ endowed with the norm $\|u\|_{X_{\alpha}}=\left\|A^{\alpha} u\right\|_{L^{2}(\Omega)}$. We note that $X_{0}=L^{2}(\Omega)$ and $X_{1}=\mathcal{D}(A)$. For two function spaces $Y$ and $Z$ satisfying $Y \subset Z$ endowed with the norm $\|\cdot\|_{Y}$ and $\|\cdot\|_{Z}$, the constant $C>0$ is referred to as the embedding constant from $Y$ to $Z$ if the following inequality holds:

$$
\sup _{u \in Y \backslash\{0\}} \frac{\|u\|_{Z}}{\|u\|_{Y}} \leq C<\infty .
$$

Note that $C$ is independent of all functions in $Y$. Furthermore, the value of $\sup _{u \in Y \backslash\{0\}}\|u\|_{Z} /\|u\|_{Y}$ is referred to as the best constant of $C$.

The main aim of this paper is to obtain the embedding constant $C_{p, \alpha}$ from $X_{\alpha}$ to $L^{p}(\Omega)$ such that

$$
\|u\|_{L^{p}(\Omega)} \leq C_{p, \alpha}\|u\|_{X_{\alpha}}, \forall u \in X_{\alpha}
$$

for $\alpha>N(1 / 2-1 / p) / 2$.

The inequality (4) is known as a Sobolev type inequality for fractional derivatives. The existence ${ }^{4}$ of the embedding constant for (4) has been studied and applied to a branch of partial differential equations $([2-7]$, etc.). The embedding constant can be used in many different ways to show the existence of solutions to partial differential equations. For example, the explicit value of the embedding constant from $H_{0}^{1}(\Omega)$ to $L^{p}(\Omega)$ plays an essential role in numerical verification of the existence of solutions to partial differential equations $[8,9]$.

If we consider (4) in $\mathbb{R}^{N}$, the best constant of the embedding constant from $X_{\alpha}$ to $L^{p}\left(\mathbb{R}^{N}\right)$ has been shown. The best constant for $p=2 N /(N-2), \alpha=1 / 2$, and $N \geq 3$ was given by Aubin [10] and Talenti [11]. Later, the best constant for $p=2 N /(N-4 \alpha)$ and $0<\alpha<N / 4$ was also obtained by Lieb [12].

Some embedding constants were obtained for the inequality (4) on the bounded domain, for example, Nakao and Yamamoto [8] derived the embedding constant for $p \in(2, \infty)$ and $\alpha=1 / 2$ using the best constant given by $[10,11]$. Xiao and Zhai $[13]$ provided a formula for the embedding constant for $2 \leq p<\infty$ and $\alpha=N / 4$ imposing some assumptions on the function $u \in X_{\alpha}$ by using the Riesz kernel and the classical Lorentz space.

For a bounded or unbounded domain with a Lipschitz boundary, Plum [9] has proposed a formula that also provides the embedding constant for $\alpha=1 / 2$. The details of these embedding constants are sketched in Section 2.

In this paper, we investigate the embedding constant on a bounded domain for $\alpha>N(1 / 2-1 / p) / 2$ using two lemmas, which are presented as in Lemma 1 and Lemma 2, with respect to the analytic semigroup $^{5} e^{-t A}$ over $L^{2}(\Omega)$ generated by $-A$. Our main theorem provides a formula for obtaining the embedding constant.

\footnotetext{
${ }^{1}$ As the inverse of the operator $A$ is a compact and self-adjoint operator, the spectral theorem shows that the operator $A$ has positive discrete spectrum (cf. [1]).

${ }^{2}$ Namely, $\lambda_{i}$ and $\psi_{i}$ satisfy $\left(\nabla \psi_{i}, \nabla v\right)_{L^{2}(\Omega)}=\lambda_{i}\left(\psi_{i}, v\right)_{L^{2}(\Omega)}, \forall v \in H_{0}^{1}(\Omega)$.

${ }^{3}$ The operator $A^{\alpha}$ is a closed and invertible operator. The closeness of $A^{\alpha}$ implies that $X_{\alpha}$ endowed with the graph norm: $\|u\|_{L^{2}(\Omega)}+\|u\|_{X_{\alpha}}$ is a Banach space. Because $A^{\alpha}$ is invertible, the graph norm is equivalent to the norm $\|u\|_{X_{\alpha}}$ (c.f. [4]).

${ }^{4}$ The existence of $C_{p, \alpha}$ for $\alpha>N(1 / 2-1 / p) / 2$ has been shown (e.g., [4]).

${ }^{5}$ We note that the operator $-A$ generates the analytic semigroup $e^{-t A}$ (c.f. [4]).
} 
Theorem 1. Let $\Omega \subset \mathbb{R}^{N}(N \in \mathbb{N})$ be a bounded domain. The minimum eigenvalue of $A$ is denoted by $\lambda_{\min }$. For $2<p \leq \infty$, let $r$ and $\alpha$ be real values such that $1 / r=1 / 2-1 / p$ and $N /(2 r)<\alpha \leq 1$, where $1 / p=0$ if $p=\infty$. Then,

$$
\|u\|_{L^{p}(\Omega)} \leq C_{p, \alpha}\|u\|_{X_{\alpha}}, \forall u \in \mathcal{D}\left(A^{\alpha}\right)
$$

holds for

$$
C_{p, \alpha}=\frac{\alpha^{\alpha} \Gamma\left(\alpha-\frac{N}{2 r}\right)}{(4 \pi)^{\frac{N}{2 r}}\left(\frac{N}{2 r}\right)^{\frac{N}{2 r}}\left(\alpha-\frac{N}{2 r}\right)^{\alpha-\frac{N}{2 r}} \Gamma(\alpha)} \lambda_{\min }^{-\left(\alpha-\frac{N}{2 r}\right)},
$$

where $\Gamma(x)$ is the Gamma function defined by $\Gamma(x)=\int_{0}^{\infty} t^{x-1} e^{-t} d t$ for $x>0$. Furthermore, if $p=2$ and $0 \leq \alpha \leq 1$, the inequality (5) also holds for

$$
C_{p, \alpha}=\lambda_{\min }^{-\alpha}
$$

Moreover, the following corollary is obtained by combining the best constant described in [12]:

Corollary 1. Let $\Omega \subset \mathbb{R}^{N}(N \in \mathbb{N})$ be a bounded domain. The minimum eigenvalue of $A$ is denoted by $\lambda_{\min }$. For $2<p<\infty$, let $r$ and $\alpha$ be real values such that $1 / r=1 / 2-1 / p$ and $N /(2 r)<\alpha \leq 1$. We impose $u=0$ on $\partial \Omega$ in the trace sense on $D\left(A^{\alpha}\right)$. Then,

$$
\|u\|_{L^{p}(\Omega)} \leq \tilde{C}_{p, \alpha}\|u\|_{X_{\alpha}}, \forall u \in \mathcal{D}\left(A^{\alpha}\right)
$$

holds for

$$
\tilde{C}_{p, \alpha}=\frac{\Gamma\left(\frac{N}{p}\right)^{\frac{1}{2}} \Gamma(N)^{\frac{1}{r}}}{(4 \pi)^{\frac{N}{2 r}} \Gamma\left(\frac{N(p-1)}{p}\right)^{\frac{1}{2}} \Gamma\left(\frac{N}{2}\right)^{\frac{1}{r}}} \lambda_{\min }^{-\left(\alpha-\frac{N}{2 r}\right)},
$$

where $\Gamma(x)$ is the Gamma function defined by $\Gamma(x)=\int_{0}^{\infty} t^{x-1} e^{-t} d t$ for $x>0$.

This paper is organized as follows: we provide some results of previous studies about embedding constants in Section 2. We prove Theorem 1 and Corollary 1 in Section 3. We present numerical examples of estimating the embedding constants including some results of previous studies in Section 4.

\section{Some previous studies related to $C_{p, \alpha}$}

Here we briefly describe previous studies of embedding constants from $X_{\alpha}$ to $L^{p}(\Omega)$. We note that $X_{1 / 2}=H_{0}^{1}(\Omega)$ and $\|u\|_{X_{1 / 2}}=\sqrt{(\nabla u, \nabla u)_{L^{2}(\Omega)}}$ hold (cf. [14]). If the domain is $\mathbb{R}^{N}(N \in \mathbb{N})$, the best constant of the Sobolev type inequality was that given by Aubin [10] and Talenti [11]. They independently derived the following estimate:

Theorem 2 ([10,11]). For $N \geq 2$, let $q$ be a real number satisfying $1<q<N$. Let $p=N q /(N-q)$. For any point $x=\left(x_{1}, \cdots, x_{N}\right) \in \mathbb{R}^{N}$, we define $|x|_{2}:=\sqrt{\left|x_{1}\right|^{2}+\cdots+\left|x_{N}\right|^{2}}$. Then,

$$
\left(\int_{\mathbb{R}^{N}}|u(x)|^{p} d x\right)^{\frac{1}{p}} \leq T_{p}\left(\int_{\mathbb{R}^{N}}|\nabla u(x)|_{2}^{q} d x\right)^{\frac{1}{q}}
$$

holds for

$$
T_{p}=\pi^{-\frac{1}{2}} N^{-\frac{1}{q}}\left(\frac{q-1}{N-q}\right)^{1-\frac{1}{q}}\left(\frac{\Gamma\left(1+\frac{N}{2}\right) \Gamma(N)}{\Gamma\left(\frac{N}{q}\right) \Gamma\left(1+N-\frac{N}{q}\right)}\right)^{\frac{1}{N}}
$$

and $T_{p}$ is the best constant of (8).

Lieb [12] also obtained the best constant as follows: 
Theorem 3 ([12]). For $N \in \mathbb{N}$, let $\alpha$ be a real number satisfying $0<\alpha<N / 4$. Then,

$$
\|u\|_{L^{\frac{2 N}{N-4 \alpha}}\left(\mathbb{R}^{N}\right)} \leq E_{\alpha}\left\|A^{\alpha} u\right\|_{L^{2}\left(\mathbb{R}^{N}\right)}, \forall u \in \mathcal{D}\left(A^{\alpha}\right)
$$

holds for

$$
E_{\alpha}=2^{-2 \alpha} \pi^{-\alpha} \sqrt{\frac{\Gamma\left(\frac{N-4 \alpha}{2}\right)}{\Gamma\left(\frac{N+4 \alpha}{2}\right)}}\left(\frac{\Gamma(N)}{\Gamma(N / 2)}\right)^{\frac{2 \alpha}{N}}
$$

and $E_{\alpha}$ is the best constant of (10).

If $\Omega$ is any bounded domain, some embedding constants were obtained. By using a zero-extension and Theorem 2, the embedding constant from $X_{1 / 2}\left(=H_{0}^{1}(\Omega)\right)$ to $L^{p}(\Omega)$ can be given as follows:

Theorem $4([8,15])$. Let $\Omega \subset \mathbb{R}^{N}(N \geq 2)$ be a bounded domain. Let $p$ be a real number such that $p \in(N /(N-1), 2 N /(N-2))$ if $N \geq 3$ and $p \in(2, \infty)$ if $N=2$. Moreover, let $q=N p /(N+p)$. Then,

$$
\|u\|_{L^{p}(\Omega)} \leq M_{p}\left\|A^{\frac{1}{2}} u\right\|_{L^{2}(\Omega)}, \forall u \in \mathcal{D}\left(A^{\frac{1}{2}}\right)
$$

holds for

$$
M_{p}=|\Omega|^{\frac{2-q}{2 q}} T_{p}
$$

where $|\Omega|$ is the measure of $\Omega$ and $T_{p}$ is a constant in (9).

Xiao and Zhai [13] obtained the following embedding constant:

Theorem 5 ([13]). Let $\Omega \subset \mathbb{R}^{N}(N \in \mathbb{N})$ be a bounded domain and $2 \leq p<\infty$. Let real numbers $r$ and $\gamma$ satisfying $1 / r=1 / 2+1 / p$ and $(1-\gamma) / r=1 / 2$. For $u \in \mathcal{D}\left(A^{N / 4}\right)$ satisfying $\operatorname{supp}\left(A^{N / 4} u\right) \subset \Omega$,

$$
\|u\|_{L^{p}(\Omega)} \leq J_{p}\left\|A^{\frac{N}{4}} u\right\|_{L^{2}(\Omega)}
$$

holds for

$$
J_{p}=\frac{N^{\frac{\gamma-1}{r}} \omega_{N-1}^{\frac{1-\gamma}{r}}|\Omega|^{\frac{\gamma}{r}}}{2^{\frac{N}{2}} \pi^{\frac{N}{2}} \gamma^{\frac{1}{r}}}
$$

where $\omega_{N-1}$ is the surface area of the unit sphere in $\mathbb{R}^{N}$.

Remark 1. Theorem 5 is obtained by substituting $q=p$ and $p=2$ into (2) of Theorem 2.1 in [13].

For cases in which the bounded or unbounded domain $\Omega$ have a Lipschitz boundary, Plum [9] provided the embedding constant using the minimum eigenvalue of $A$.

Theorem 6 ([9]). Let $\lambda_{\min }$ be the minimum eigenvalue of the Laplace operator for $\Omega \subset \mathbb{R}^{N}(N \geq$ 2) with a Lipschitz boundary. Specify $p \in[2,2 N /(N-2)))$ and $s=N(1 / p-1 / 2+1 / N)$, where $2 N /(N-2)=\infty$ if $N=2$. Then,

$$
\|u\|_{L^{p}(\Omega)} \leq L_{p}\left\|A^{\frac{1}{2}} u\right\|_{L^{2}(\Omega)}, \forall u \in \mathcal{D}\left(A^{\frac{1}{2}}\right)
$$

holds for

$$
L_{p}= \begin{cases}\left(\frac{1}{2}\right)^{\frac{1}{2}+\frac{2 \nu-3}{p}}\left[\frac{p}{2}\left(\frac{p}{2}-1\right) \cdots\left(\frac{p}{2}-\nu+2\right)\right]^{\frac{2}{p}} \lambda_{\min }^{-\frac{1}{p}} & (N=2), \\ \left(\frac{N-1}{\sqrt{N}(N-2)}\right)^{1-s} \lambda_{\text {min }}^{-\frac{s}{2}} & (N \geq 3),\end{cases}
$$

respectively, where $\nu$ is the maximum integer such that $\nu \leq p / 2$ and the term in brackets is 1 if $\nu=1$ and $N=2$. 


\section{Proofs of Theorem 1 and Corollary 1}

We introduce two fundamental lemmas in order to prove Theorem 1 and Corollary 1 . The following lemma holds by using the fundamental theory of a semigroup:

Lemma 1 (cf. $[4,14])$. Let $\Omega \subset \mathbb{R}^{N}(N \in \mathbb{N})$ be a bounded domain. For $0 \leq \beta \leq 1, A^{\beta}: \mathcal{D}\left(A^{\beta}\right) \rightarrow$ $L^{2}(\Omega)$ is invertible and

$$
\left(A^{\beta}\right)^{-1} u=\Gamma(\beta)^{-1} \int_{0}^{\infty} t^{\beta-1} e^{-t A} u d t
$$

is expressed for $u \in L^{2}(\Omega)^{6}$.

Moreover, some properties of the Dirichlet heat kernel give the following lemma:

Lemma 2 (cf. [16]). Let $\Omega \subset \mathbb{R}^{N}(N \in \mathbb{N})$ be a bounded domain. For $1 \leq p<q \leq \infty$, put $1 / r=1 / p-1 / q$, where $1 / q=0$ if $q=\infty$. For all $t \in(0, \infty)$,

$$
\left\|e^{-t A} u\right\|_{L^{q}(\Omega)} \leq(4 \pi t)^{-\frac{N}{2 r}}\|u\|_{L^{p}(\Omega)}, \forall u \in L^{p}(\Omega)
$$

holds.

For $0 \leq \alpha<1, A^{-\alpha}$ denotes $\left(A^{\alpha}\right)^{-1}$. For any bounded operator $T: L^{p}(\Omega) \rightarrow L^{q}(\Omega)(1 \leq p, q \leq \infty)$, let

$$
\|T\|_{L^{p}, L^{q}}=\sup _{u \in L^{p}(\Omega) \backslash\{0\}} \frac{\|T u\|_{L^{q}(\Omega)}}{\|u\|_{L^{p}(\Omega)}} .
$$

First, we prove Theorem 1.

Proof of Theorem 1. First, we show that Theorem 1 holds for $2<p \leq \infty$. Let $r$ and $\alpha$ be real values such that $1 / r=1 / 2-1 / p$ and $N /(2 r)<\alpha \leq 1$, where $1 / p=0$ if $p=\infty$. Put $u \in \mathcal{D}\left(A^{\alpha}\right)$. From Lemma 1,

$$
\begin{aligned}
\|u\|_{L^{p}(\Omega)} & =\left\|A^{-\alpha} A^{\alpha} u\right\|_{L^{p}(\Omega)} \\
& \leq \Gamma(\alpha)^{-1} \int_{0}^{\infty} t^{\alpha-1}\left\|e^{-t A} A^{\alpha} u\right\|_{L^{p}(\Omega)} d t \\
& \leq \Gamma(\alpha)^{-1} \int_{0}^{\infty} t^{\alpha-1}\left\|e^{-t A}\right\|_{L^{2}, L^{p}}\left\|A^{\alpha} u\right\|_{L^{2}(\Omega)} d t \\
& \leq \Gamma(\alpha)^{-1} \int_{0}^{\infty} t^{\alpha-1}\left\|e^{-\beta t A}\right\|_{L^{2}, L^{p}}\left\|e^{-(1-\beta) t A}\right\|_{L^{2}, L^{2}}\left\|A^{\alpha} u\right\|_{L^{2}(\Omega)} d t
\end{aligned}
$$

holds for $0<\beta<1$. The spectral mapping theorem and Lemma 2 state that

$$
\begin{aligned}
\|u\|_{L^{p}(\Omega)} & \leq \Gamma(\alpha)^{-1} \int_{0}^{\infty} t^{\alpha-1}(4 \pi \beta t)^{-\frac{N}{2 r}} e^{-t(1-\beta) \lambda_{\min }}\left\|A^{\alpha} u\right\|_{L^{2}(\Omega)} d t \\
& =(4 \pi \beta)^{-\frac{N}{2 r}} \Gamma(\alpha)^{-1} \int_{0}^{\infty} t^{\alpha-1-\frac{N}{2 r}} e^{-t(1-\beta) \lambda_{\min }} d t\left\|A^{\alpha} u\right\|_{L^{2}(\Omega)} \\
& =(4 \pi \beta)^{-\frac{N}{2 r}} \Gamma(\alpha)^{-1}\left(\frac{1}{(1-\beta) \lambda_{\min }}\right)^{\alpha-1-\frac{N}{2 r}} \int_{0}^{\infty} s^{\alpha-1-\frac{N}{2 r}} e^{-s}\left(\frac{1}{(1-\beta) \lambda_{\min }}\right) d s\left\|A^{\alpha} u\right\|_{L^{2}(\Omega)} \\
& =\frac{\Gamma\left(\alpha-\frac{N}{2 r}\right)}{(4 \pi)^{\frac{N}{2 r}} g(\beta) \Gamma(\alpha)} \lambda_{\min }^{-\left(\alpha-\frac{N}{2 r}\right)}\left\|A^{\alpha} u\right\|_{L^{2}(\Omega)}
\end{aligned}
$$

holds, where $g(\beta):=\beta^{\frac{N}{2 r}}(1-\beta)^{\alpha-\frac{N}{2 r}}(0<\beta<1)$ and $\Gamma(\alpha-N / 2 r)<\infty$ from $\alpha>N /(2 r)$. Because the function $g$ admits the maximal value at $\beta=\frac{N}{2 r \alpha}(<1)$, it follows that

$$
\|u\|_{L^{p}(\Omega)} \leq \frac{\alpha^{\alpha} \Gamma\left(\alpha-\frac{N}{2 r}\right)}{(4 \pi)^{\frac{N}{2 r}}\left(\frac{N}{2 r}\right)^{\frac{N}{2 r}}\left(\alpha-\frac{N}{2 r}\right)^{\alpha-\frac{N}{2 r}} \Gamma(\alpha)} \lambda_{\min }^{-\left(\alpha-\frac{N}{2 r}\right)}\left\|A^{\alpha} u\right\|_{L^{2}(\Omega)} .
$$

\footnotetext{
${ }^{6}$ The function $\left(A^{\beta}\right)^{-1} u$ can be expressed by using the Dunford integral (e.g., [14]). The resulting expression corresponds with the right hand of (16) (e.g., [4]).
} 
Next, we prove Theorem 1 for $p=2$. For $0 \leq \alpha \leq 1$ and $u \in \mathcal{D}\left(A^{\alpha}\right)$, the spectral mapping theorem and Lemma 1 yield

$$
\begin{aligned}
\|u\|_{L^{2}(\Omega)} & =\left\|A^{-\alpha} A^{\alpha} u\right\|_{L^{2}(\Omega)} \\
& \leq \Gamma(\alpha)^{-1} \int_{0}^{\infty} t^{\alpha-1}\left\|e^{-t A} A^{\alpha} u\right\|_{L^{2}(\Omega)} d t \\
& \leq \Gamma(\alpha)^{-1} \int_{0}^{\infty} t^{\alpha-1}\left\|e^{-t A}\right\|_{L^{2}, L^{2}} d t\left\|A^{\alpha} u\right\|_{L^{2}(\Omega)} \\
& \leq \Gamma(\alpha)^{-1} \int_{0}^{\infty} t^{\alpha-1} e^{-t \lambda_{\min }} d t\left\|A^{\alpha} u\right\|_{L^{2}(\Omega)} \\
& =\lambda_{\min }^{-\alpha}\left\|A^{\alpha} u\right\|_{L^{2}(\Omega)} .
\end{aligned}
$$

From (19), $\left\|A^{-\alpha}\right\|_{L^{2}, L^{2}} \leq \lambda_{\min }^{-\alpha}$ and $C_{2, \alpha}=\lambda_{\min }^{-\alpha}$ hold for $0 \leq \alpha \leq 1$.

Next, we provide the proof of Corollary 1 by using Theorem 3 and (19).

Proof of Corollary 1. For $\beta=N(p-2) / 4 p(<N / 4)$, let $E_{\beta}$ be defined by (11). As a results of extension by zero and Theorem 3 , it follows for $u \in \mathcal{D}\left(A^{\alpha}\right)$

$$
\begin{aligned}
\|u\|_{L^{p}(\Omega)} & =\|u\|_{L^{p}\left(\mathbb{R}^{N}\right)} \\
& \leq E_{\beta}\left\|A^{\beta} u\right\|_{L^{2}\left(\mathbb{R}^{N}\right)} \\
& =E_{\beta}\left\|A^{\beta} u\right\|_{L^{2}(\Omega)}
\end{aligned}
$$

where $\beta=N(p-2) / 4 p=N / 2 r<\alpha$ and $\mathcal{D}\left(A^{\alpha}\right) \subset \mathcal{D}\left(A^{\beta}\right)$ for $\beta<\alpha$ (cf. [4]). Moreover, (19) gives

$$
\begin{aligned}
\|u\|_{L^{p}(\Omega)} & \leq E_{\beta}\left\|A^{\beta-\alpha}\right\|_{L^{2}, L^{2}}\left\|A^{\alpha} u\right\|_{L^{2}(\Omega)} \\
& \leq E_{\beta} \lambda_{\min }^{\beta-\alpha}\left\|A^{\alpha} u\right\|_{L^{2}(\Omega)}
\end{aligned}
$$

The inequality (20) implies that Corollary 1 holds.

Remark 2. All elements $u \in X_{\alpha}$ do not always satisfy $u=0$ on $\partial \Omega$ in the trace sense. For example, we consider the regularity of functions in $X_{\alpha}$ if $\Omega$ is bounded and convex. Then, it is well known that the function space $X_{\alpha}$ is equivalent to the fractional Sobolev space $H^{2 \alpha}(\Omega)$ for $0 \leq \alpha<1 / 4$ [17]. Note that all elements $u \in X_{\alpha}$ satisfy $u=0$ on $\partial \Omega$ in the trace sense for $1 / 4<\alpha \leq 1$ and $\alpha \neq 3 / 4$ [17]. Moreover, if $\Omega \subset \mathbb{R}^{2}$ is a convex polygon, it is proved that all elements $u \in X_{\alpha}$ satisfy $u=0$ on $\partial \Omega$ even for $\alpha=3 / 4$ [18].

\section{Numerical examples}

In this section, we provide some numerical examples to estimate the embedding constant $C_{p, \alpha}$ in Theorem 1 and $\tilde{C}_{p, \alpha}$ in Corollary 1. All computations were carried out on computer running Windows 7 Professional with an Intel (R) Core (TM) i7-5600U CPU and 16GB RAM. We used MATLAB R2012a with INTLAB ver. 7.1 [19]. Let $\Omega:=(0,1) \times(0,1)$ and $\alpha=1 / 2$. We note $\lambda_{\min }=2 \pi^{2}$. We computed $C_{p, 1 / 2}$ in Theorem 1, $\tilde{C}_{p, 1 / 2}$ in Corollary $1, M_{p}$ in Theorem $4, J_{p}$ in Theorem 5 , and $L_{p}$ in Theorem 6 , respectively. The values of these constants are displayed in Table I.

In Table I, $C_{p, 1 / 2}$ is a rough estimate compared with the other estimates. However, $\tilde{C}_{p, 1 / 2}$ is tighter than the other values except for $M_{p}$.

Varying $p=3,4,5,6$, and $\alpha$ such that $1 / 2-1 / p<\alpha \leq 1, C_{p, \alpha}$ in Theorem 1 and $\tilde{C}_{p, \alpha}$ in Corollary 1 are plotted on the domain $\Omega=(0,1) \times(0,1)$ in Figs. 1, 2, 3, and 4 , respectively. The plots in the four figures indicate that the estimate in Corollary 1 is sharper than that in Theorem 1. However, as can be seen in Fig. 1, $\tilde{C}_{p, \alpha}$ is not plotted for $1 / 6<\alpha<1 / 4$ because the estimate in Corollary 1 does not hold for $\alpha<1 / 4$ (e.g., Remark 2 of this paper).

Let $\Omega=(0,2) \times(0,2) \backslash[1,2] \times[1,2]$ and $\alpha=1 / 2$. Then, the minimum eigenvalue over the domain $\Omega$ is included in [9.639717, 9.639724] [20]. We compute $C_{p, 1 / 2}, \tilde{C}_{p, 1 / 2}, M_{p}, J_{p}$, and $L_{p}$, respectively. The value of these constants are displayed in Table II. Similar to the results in Table I, $C_{p, \alpha}$ is the 
Table I. Comparison of each value on the domain $\Omega=(0,1) \times(0,1)$.

\begin{tabular}{c|c|c|c|c|c}
\hline$p$ & $C_{p, 1 / 2}$ & $\tilde{C}_{p, 1 / 2}$ & $M_{p}$ & $J_{p}$ & $L_{p}$ \\
\hline 3 & 0.504227914 & 0.298833496 & 0.279911047 & 0.605357242 & 0.329648994 \\
\hline 4 & 0.728930690 & 0.356352736 & 0.318309887 & 0.643037069 & 0.398942281 \\
\hline 5 & 0.934611867 & 0.406084557 & 0.357803885 & 0.678020304 & 0.489090310 \\
\hline 6 & 1.129584278 & 0.450720364 & 0.395853999 & 0.710834333 & 0.552669458 \\
\hline
\end{tabular}

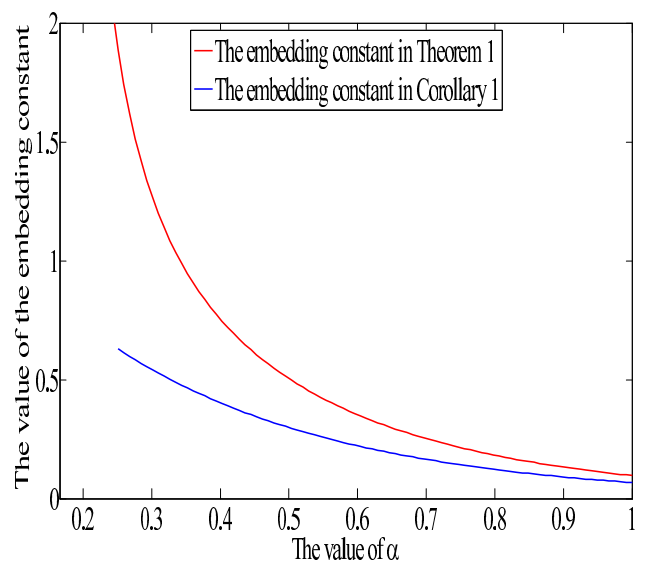

Fig. 1. Values of $C_{3, \alpha}$ and $\tilde{C}_{3, \alpha}$ on the domain $\Omega=(0,1) \times(0,1)$.

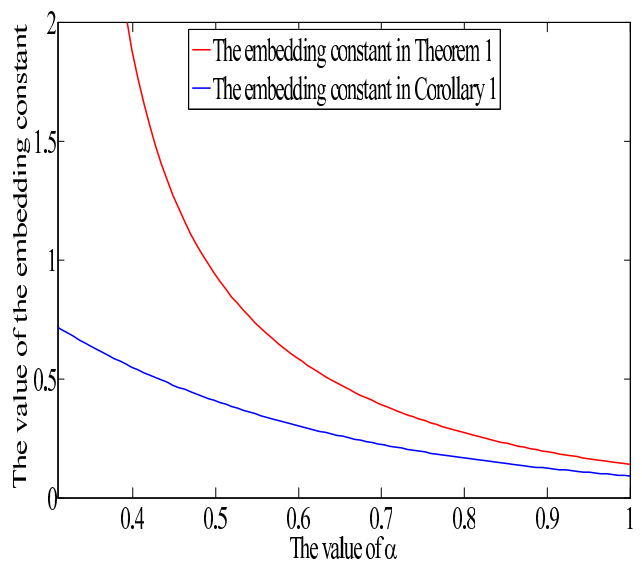

Fig. 3. Values of $C_{5, \alpha}$ and $\tilde{C}_{5, \alpha}$ on the domain $\Omega=(0,1) \times(0,1)$.

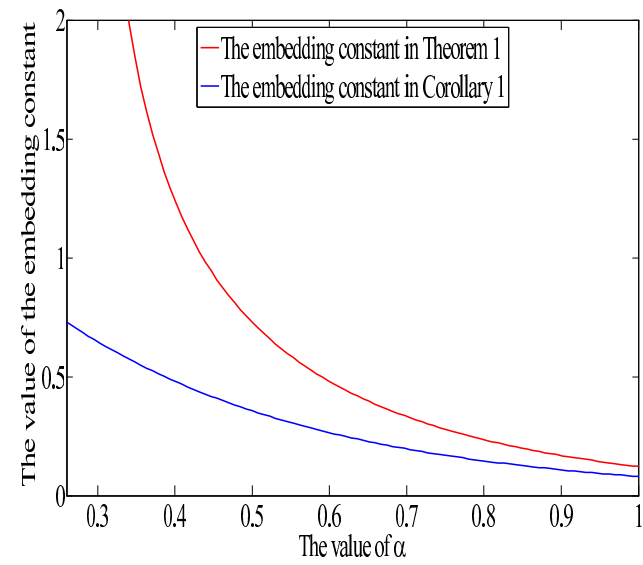

Fig. 2. Values of $C_{4, \alpha}$ and $\tilde{C}_{4, \alpha}$ on the domain $\Omega=(0,1) \times(0,1)$.

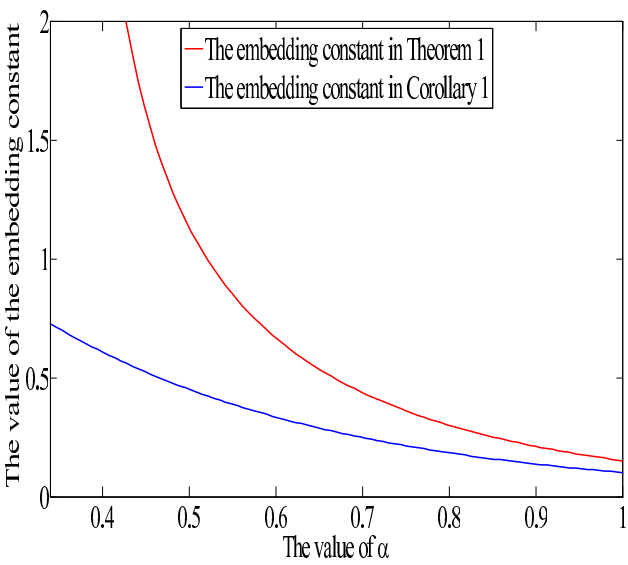

Fig. 4. Values of $C_{6, \alpha}$ and $\tilde{C}_{6, \alpha}$ on the domain $\Omega=(0,1) \times(0,1)$.

Table II. Comparison of each value on the domain $\Omega=(0,2) \times(0,2) \backslash[1,2] \times$ $[1,2]$.

\begin{tabular}{c|c|c|c|c|c}
\hline$p$ & $C_{p, 1 / 2}$ & $\tilde{C}_{p, 1 / 2}$ & $M_{p}$ & $J_{p}$ & $L_{p}$ \\
\hline 3 & 0.640297840 & 0.379476099 & 0.403701587 & 0.8730762213 & 0.418607405 \\
\hline 4 & 0.871972121 & 0.426281476 & 0.418919370 & 0.8462843754 & 0.477228565 \\
\hline 5 & 1.078659524 & 0.468672602 & 0.445727370 & 0.8446308696 & 0.564471670 \\
\hline 6 & 1.272905652 & 0.507907653 & 0.475395696 & 0.8536672189 & 0.622792022 \\
\hline
\end{tabular}

rough estimate and the value of $\tilde{C}_{p, \alpha}$ is tighter than the other constants except for $M_{p}$. Varying $p=3,4,5,6$ and $\alpha$ such that $1 / 2-1 / p<\alpha \leq 1, C_{p, \alpha}$ in Theorem 1 are plotted on the domain $\Omega=(0,2) \times(0,2) \backslash[1,2] \times[1,2]$ in Figs. 5, 6, 7, and 8, respectively. On the other hand, the values of $\tilde{C}_{p, \alpha}$ are not plotted. This is because the domain $\Omega$ is a non-convex domain; therefore, it is difficult for us to judge the range of $\alpha$ in which all elements $u \in \mathcal{D}\left(A^{\alpha}\right)$ satisfy $u=0$ on $\partial \Omega$ in the trace sense.

Moreover, we recall that our main theorem enables us to obtain the embedding constant from $X_{\alpha}$ to $L^{\infty}(\Omega)$ for $\alpha>1 / 2$ by Theorem 1 . Figures 9 and 10 show the embedding constant $C_{\infty, \alpha}$ in Theorem 1 


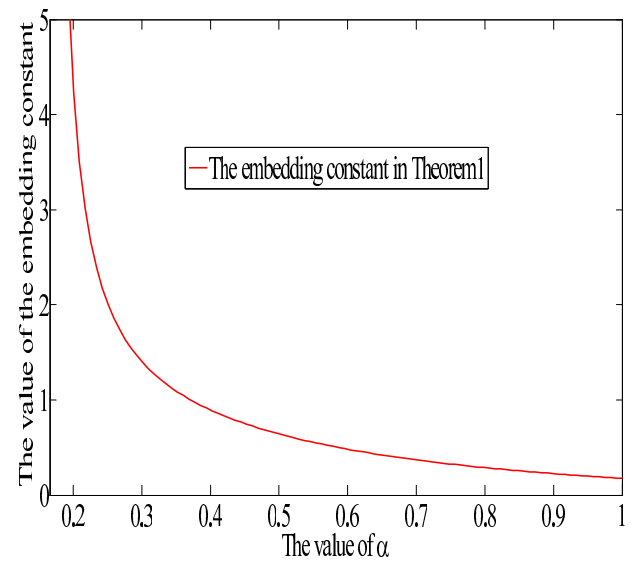

Fig. 5. Values of $C_{3, \alpha}$ on the domain $\Omega=(0,2) \times(0,2) \backslash[1,2] \times[1,2]$.

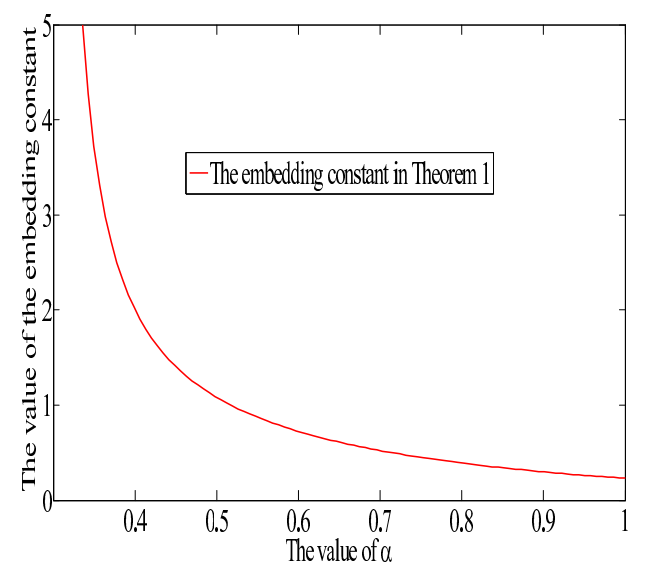

Fig. 7. Values of $C_{5, \alpha}$ on the domain $\Omega=(0,2) \times(0,2) \backslash[1,2] \times[1,2]$.

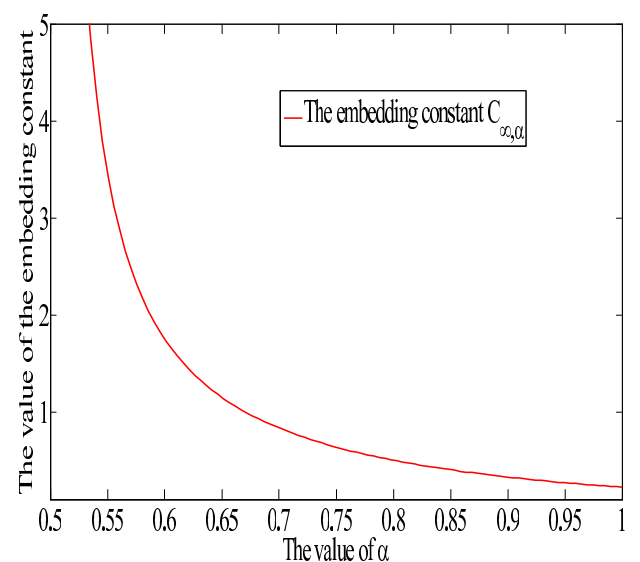

Fig. 9. Values of $C_{\infty, \alpha}$ on the domain $\Omega=(0,1) \times(0,1)$.

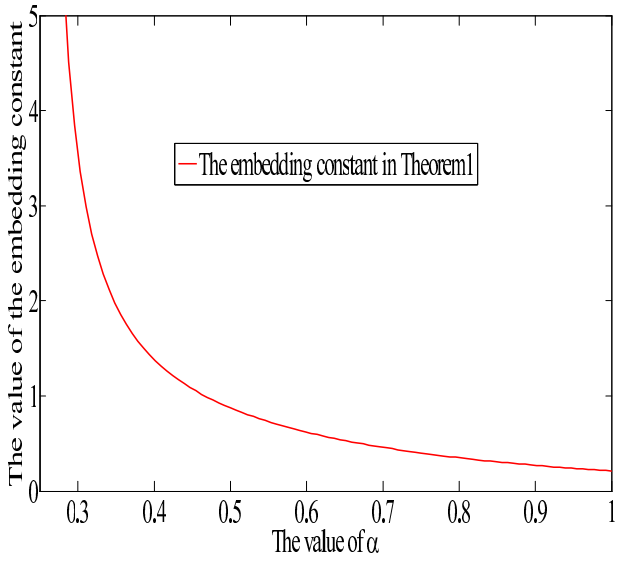

Fig. 6. Values of $C_{4, \alpha}$ on the domain $\Omega=(0,2) \times(0,2) \backslash[1,2] \times[1,2]$.

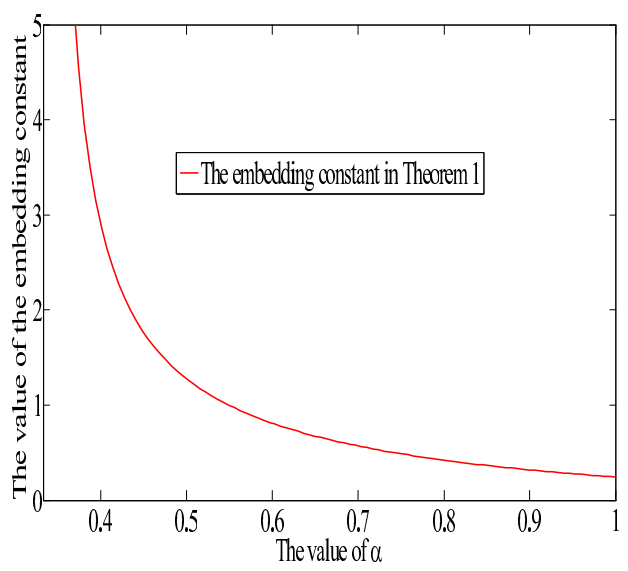

Fig. 8. Values of $C_{6, \alpha}$ on the domain $\Omega=(0,2) \times(0,2) \backslash[1,2] \times[1,2]$.

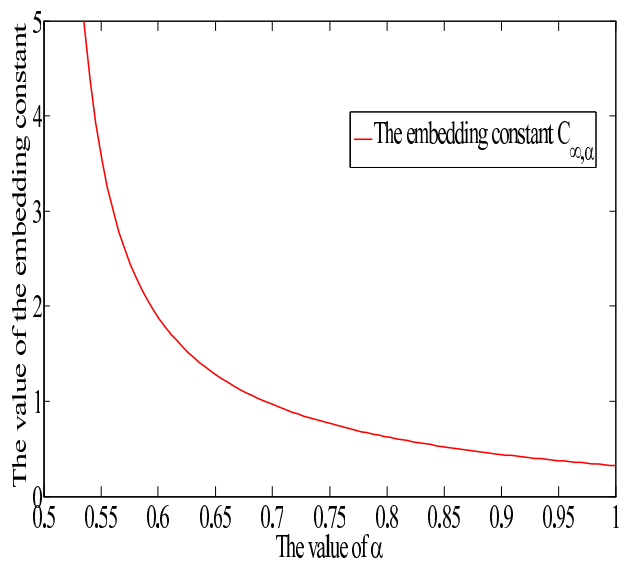

Fig. 10. Values of $C_{\infty, \alpha}$ on the domain $\Omega=(0,2) \times(0,2) \backslash[1,2] \times[1,2]$.

for $1 / 2<\alpha \leq 1$ on $\Omega=(0,1) \times(0,1)$ and $\Omega=(0,2) \times(0,2) \backslash[1,2] \times[1,2]$, respectively. The results in these two figures indicate that each embedding constant $C_{\infty, \alpha}$ seems to grow up if $\alpha$ tends to $1 / 2$, respectively. Note that we cannot obtain the embedding constant $X_{\alpha}$ to $L^{\infty}(\Omega)$ using Corollary 1.

\section{Acknowledgments}

The authors wish to express their thanks to Prof. Norikazu Saito from the University of Tokyo for his comments that originally led them to embark upon this study. The authors would also like express their gratitude to Dr. Kouta Sekine, and Mr. Kazuaki Tanaka from Waseda University for their 
valuable comments and kind remarks, and to anonymous referees for providing useful comments to improve this paper. This work was supported in part by JSPS Grant-in-aid for Scientic Research (C), No. 15K04946 and JSPS Grant-in-Aid for Young Scientists (B), No. 15K17596, respectively. We would like to thank Editage (www.editage.jp) for English language editing.

\section{References}

[1] R. Dautray, J. Lions, M. Artola, and M. Cessenat, Mathematical Analysis and Numerical Methods for Science and Technology: Spectral Theory and Applications, Springer-Verlag, New York, 2000.

[2] J.L. Lions and E. Magenes, Non-homogeneous boundary value problems and applications, Springer Berlin Heidelberg, 1972.

[3] R.A. Adams, Sobolev spaces, Second ed., Academic Press, New York, 1975.

[4] A. Pazy, Semigroup of linear operators and applications to partial differential equations, Springer, 1983.

[5] A. Cotsiolis and N.K. Tavoularis, "Best constants for Sobolev inequalities for higher order fractional derivatives," J. Math. Anal. Appl., vol. 295, pp. 225-236, July 2004.

[6] G.A. Anastassiou, "Fractional Sobolev type inequalities," Applicable Analysis, vol. 87, no. 5, pp. 607-624, May 2008.

[7] A. Aibeche, W. Chikouche, and Y. Daikh, "Real interpolation spaces between the domain of the laplace operator with transmission conditions and $L^{p}$ on a polygonal domain," Electronic Journal of Differential Equations, vol. 2012, no. 10, pp. 1-14, January 2012.

[8] M.T. Nakao and N. Yamamoto, Numerical verification, Nihonhyouron-sya, 1988. (in Japanese)

[9] M. Plum, "Existence and multiplicity proofs for semilinear elliptic boundary value problems by computer assistance," DMV Jahresbericht: JB 110, pp. 19-54, 2008.

[10] T. Aubin, "Problémes isopérimetriques et spaces de Sobolev," Journal of Differential Geometry, vol. 11, pp. 573-598, 1976. (in French)

[11] G. Talenti, "Best constant in Sobolev inequality," Annali di Matematica pura ed Applicata, vol. 110, pp. 353-372, 1976.

[12] E.H. Lieb, "Sharp constants in the Hardy-Littlewood-Sobolev inequalities and related inequalities," Annals of Mathematics., vol. 118, pp. 349-374, 1983.

[13] J. Xiao and Zh. Zhai, "Fractional Sobolev, Moser-Trudinger, Morrey-Sobolev inequalities under lorentz norms," Journal of Mathematical Sciences, vol. 166, no. 3, pp. 357-376, 2010.

[14] A. Yagi, Abstract parabolic evolution equations and their applications, Springer-Verlag, Berlin Heidelberg, 2010.

[15] K. Tanaka, K. Sekine, M. Mizuguchi, and S. Oishi, "Numerical method for deriving sharp inclusion of the Sobolev embedding constant on bounded convex domain," arXiv:1503.05468, http://arxiv.org/abs/1503.05468.

[16] P. Quittner and P. Souplet, Superlinear parabolic problems blow-up, global existence and steady states, Birkhäuser-Verlag, Basel, 2007.

[17] A. Yagi, " $H_{\infty}$ functional calculus and characterization of domains of fractional powers," Operator Theory: Advances and Applications, vol. 187, pp. 217-235, 2008.

[18] N. Saito, "On interpolation spaces in a polygon," Memories of the Faculty of Human development University of Toyama, vol. 2, no. 1, pp. 7-12, 2007.

[19] S.M. Rump, "INTLAB-INTerval LABoratory," in Developments in Reliable Computing, ed. Tibor Csendes, Kluwer, pp. 77-104, Kluwer Academic Publisher, Dordrecht, 1999.

[20] X. Liu, T. Okayama, and S. Oishi, "High-Precision Eigenvalue Bound for the Laplacian with Singularities," Computer Mathematics, pp. 311-323, Springer-Verlag Berlin Heidelberg, 2014. 